\title{
Jeffrey Michael Isner 1947-2001
}

Teffrey Michael Isner's death on 29 $\int$ October cut short his career at the age of 53, but not before he inspired numerous scientists with his innovative research and raised the hopes of many patients with cardiovascular disease. The sad news of his untimely passing was shocking. Jeffrey died unexpectedly of a heart attack, the very disease that, as a cardiologist, he was committed to curing.

During his all too brief but successful scentific career, Isner's inquiring mind, keen observational powers and thirst for the unknown led to key discoveries of the events surrounding angiogenesis-the formation of new blood vessels. He pioneered efforts to use gene therapy to introduce a key angiogenic factor, vascular endothelial

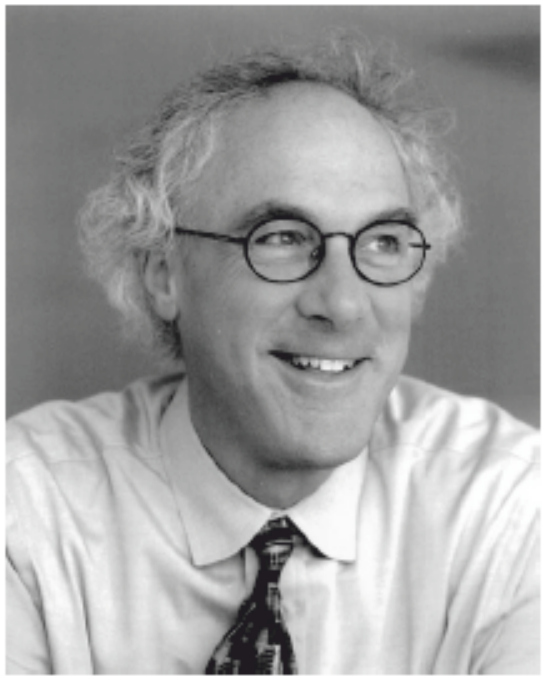

matter of concern considering that VEGF is a potent molecule and that angiogenesis stimulates cancer and blindness in diabetes.

Encouraged by his success in preclinical studies, he pioneered VEGF clinical trials to treat patients with myocardial or ischemic limb disease and reported the first clinical results in 1998 . These data were encouraging and have prompted more extensive studies to identify optimal approaches.

But his work was not all smooth sailing. In 1999, gene therapy trials faced a setback with the death of a teenager following injection of a genetically altered virus (Nature Med. 6, 6; 2000). Two years after creating a private company to develop his growth factor (VEGF), into the heart and limb to coax blood vessels to grow-a process for which he coined the phrase 'therapeutic angiogenesis'. As a cardiologist, Isner was driven by the potential that this therapy offered for patients with cardiovascular disease. In the latter part of his career, his work led to the identification of endothelial precursors, which he intended to use to grow new blood vessels.

He earned his MD in 1973 at Tufts Untversity School of Medicine, Boston, and trained as a cardiologist at Georgetown University Hospital, Washington, DC. After a decade of clinically oriented research, he became intrigued in the early 1990 s by the potential to transfer genes into the walls of blood vessels and the heart. Primed by the seminal work of Gary and Elizabeth Nabel in delivering genes to the vasculature, Isner recognized that this technology would allow him to develop novel therapeutic avenues for treating patients with inoperable heart disease and limb ischemia. This would become an enduring theme to which he devoted the rest of his career.

Isner pioneered efforts and optimized techniques to transfer naked plasmid DNA of therapeutic genes. This strategy is relatively safe, manageable for dally clinical use and, compared to its relative inefficiency in other cell types, unexpectedly more effective in ischemic muscle. In part, thanks to Isner's perseverance, half of the clinical trials now in the cardiovascular portfolio employ either naked DNA or liposome carriers.

His scientific career can be tracked through hundreds of publications, including several in Nature Medicine. In one of his marny papers, he commented that "despite the low transfection efficiency of naked plasmid DNA, " the success of VEGF gene therapy for therapeutic angiogenesis can be ascribed to the fact that "VEGF is naturally secreted [and therefore can affect neighboring non-transfected cells], binds to high-affinity receptors that are upregulated by hypoxia, and stimulates endothelial survival".

Isner argued that "transient gene expression would be sufficient to promote neovascularization, whereas persistent flow, rather than persistent gene expression, would constitute the principal determinant of subsequent vessel durability". In addition, "compared with protracted gene expression, short-term VEGF gene expression would be less toxic and safer." This is a research, Isner found himself in the midst of debates among researchers and regulators about the safety of gene therapy, financlal conflicts of interest and patient protection, and his clinical gene therapy program was suspended by the FDA.

Isner persisted under close scrutiny, and last November the FDA granted him permission to resume one of the three halted trials. His efforts were aided by a private donation of $\$ 1$ million to St. Elizabeth's Hospital, and subsequently by a $\$ 10$ million NIH grant-direct evidence of support and belief in Isner's ability to make major progress in angiogenesis research. Only a few months ago, the National Heart Lung and Blood Institute awarded Isner a 'Center of Excellence' award.

His contributions went well beyond VEGF gene therapy. Another breakthrough in his career was heralded by his report in 1997 of the isolation of putative progenitor endothelial cells for angiogenesis. Developmental biologists had suspected the existence of endothelial stem cells in the embryo, but their identification in adult bone marrow was a novel and provocative finding. It drew attention from many vascular biologists and cardiologists because of the therapeutic potential to regenerate new vessels in ischemic tissues. Isner and coworkers demonstrated that these endothelial progenitors were mobilized to sites of angiogenesis, contributed to the formation of new vessels and improved perfusion of ischemic tissue. He believed that the future promise for therapeutic angiogenesis lay in combining endothelial stem-cell-based therapy with angiogenic growth factor gene therapy.

Jeffrey was a caring doctor, an inspiring mentor and a man who created an internationally recognized cardiovascular research program. He dedicated his life to medical science but passed away leaving some questions unanswered. Yet, he paved the route for many others to follow, a legacy that will remain vibrant and lasting for many more years to come.

PETER CARMeIIET Center for Transgene Technology and Gene Therapy Fanders Interuniversity Institute for Biotechnology University of Leuven, Leuven, Belgium Email: peter.carmeliat đmed.kyleuven.ac.be 